\title{
Our 21st century health identity: Where is sleep?
}

\author{
Karen Spruyt
}

Published online: 13 February 2014

(C) Springer-Verlag Berlin Heidelberg 2014

That public health affects $u s$ is well documented in this issue of the Journal of Public Health. The topics in this issue cover health across the lifespan with six manuscripts discussing in detail the promotion of health of the most vulnerable people; i.e., children, women and minorities. Several dimensions of their health are adversely affected, and the interdisciplinary approach to their health discussed in this issue underscores the importance of a pertinent network of public health worldwide. This is especially the case when vulnerability 'accumulates', forming our 21 st century health identity. More specifically, a worldwide public health network should not only govern threats to health during the daytime but equally during nighttime. For where we sleep, how we sleep, when we sleep, how much we sleep... or the "sleep-determinants of health" are amongst the many factors affecting our health in general. Thus, our current health, or the health of young adults and the elderly, as well as our future health or our children's health, should be subject to discussion and debate from a day/night perspective.

Whether guidelines are implemented in real life, health care is a highly relevant topic in terms of continued public health education and training of professionals as well as in providing optimum health care. Several reasons for not adhering to guidelines, such as reduced vigilance during our professional

\footnotetext{
K. Spruyt ( $\square)$

Faculty of Psychology and Educational Sciences, Vrije Universiteit Brussel, Pleinlaan 2, Brussel 1050, Belgium

e-mail: spruytsleep@gmail.com

\section{K. Spruyt}

Department of Developmental and Behavioral Pediatrics, Shanghai Children's Medical Center affiliated with Shanghai Jiaotong

University School of Medicine, Shanghai, China

K. Spruyt

Maastricht University Medical Center, Rett Expertise

Centre - Governor Kremers Centre, Maastricht, The Netherlands
}

endeavors, can be listed. Sleep studies in medical residents suggest impairments in several domains, including attention, cognition, motor skills, and mood (Mansukhani et al. 2012; Reed et al. 2010). Unfortunately, in such a global fast-paced lifestyle, only medical errors or complications may alert us. In their observational study, Boettger et al. recommend improvements in the treatment of patients with impaired renal function. The authors discuss the practice of therapy in these patients and alert us to the negative clinical implications and the legal reasons.

Being attentive regarding risk factors for adverse health outcomes can extend to the general population. In adults aged $50+$, behavioral risk factors are likely multiple and varied. Linardakis et al. indicate that across European countries, the prevalence rate of multiple behavioral risk factors such as alcohol, smoking habits, physical inactivity is different. Regarding all risk factors studied, inactivity prevailed; thus, one may question the association of inactivity with sleep (Ding et al. 2013). Yet, already at a young age, behavioral risks may accrue; for instance, poor sleep (Spruyt et al.) may further promote health threats. Difficulties initiating or maintaining sleep, or circumstances impeding optimal sleep may increase vulnerability to adverse health outcomes (Spruyt and Gozal 2012; Spruyt et al. 2011).

Alternatively, sleep problems such as difficulties staying asleep is commonly reported in subjects with posttraumatic stress disorder (Lamarche and De Koninck 2007; Singareddy and Balon 2002). Haase et al., for instance, report on human trafficking, a violation of human dignity affecting mostly women and children. The report is regarding the three Pparadigm - prevention, protection and prosecution —which was developed by a highly disciplinary group under the framework of the Siena Principles on Human Trafficking and Public Health. The authors emphasize the substantial health and social implications, with long-lasting grave and adverse impact on the individual, public health, human 
security as well as human development caused by human trafficking. Additionally, along with the medical, social, and legal implications of trafficking are potential medical risksnamely, in contrast to developed countries, in tropical and developing countries, other health problems such as typhoid fever may exist. Senthilkumar et al. compare Toll Like Receptor 5 (TLR5) polymorphism and various clinical parameters in typhoid patients and asymptomatic typhoid carriers, concluding with the important regulating role of TLRs towards innate and adaptive immunity.

Another example of stress relates to single parenthood. Weihrauh et al. show that an attachment-based, emotioncentered parental training program can be effective in reducing depressive symptoms as well as the psychological symptom load, and it can enhance the psychological well-being of moderately burdened single mothers with preschool-aged children. In terms of sleep, this could translate into success on both sides - improved sleep for both the mother and child (Bagley and El-Sheikh 2013; Keller et al. 2013).

Although trigger factors to suicide or self-harm can be highly diverse, sleep disturbances such as insomnia and nightmares have been associated with both (Pigeon et al. 2012). Ngwena et al. show, more specifically, that in an inner city, suicide and undetermined injury among United Kingdom born black and minority ethnic groups, followed by East and West Europeans, was higher than the national average. Associations of insufficient sleep with suicide attempts and selfinjury in a large sample of adolescents has been put forward (Kang et al. 2013).

Adolescence is a (often stressful) transitional stage characterized by substantial developmental changes, one of which involves the ability to make autonomous decisions about one's sexual life within the context of one's own personal and social ethics. Ju-Eun Song et al. describe the improvement in contraception knowledge, attitudes, and sexual autonomy among university students through a sexual autonomy enhancement program developed according to the main concepts of the health belief model. Healthy decision-making, thus lowering behavioral health risk factors, starts early in life.

Sleep researchers are continuously faced with the difficult task to make sleep 'a trendy behavior' or, alternatively, to define which type of message will promote sleep. A similar quest is outlined by Borzekowski et al.; namely, how to reach young children with health messages given their developing abilities regarding information processing. Their study showed that health warning labels featured on cigarette packages are not effectively reaching 5 - and 6-year-old children.
Also, the last manuscript in this issue discusses sleepoverlapping health-related risk factors: physical inactivity, obesity and hypertension. High body fat and low levels of physical activity were found to be cardiovascular risk factors in a study by Poethko-Müller et al. using a submaximal cycle ergometer test. They further discuss the intriguing association of these factors with social inequality and migration background.

Although most studies discussed in this issue of the Journal of Public Health focus on day-time health issues, dear to our heart remains sleep. We believe that sleep can be a health promoter, individually and publically, and advocate that the waking-state cannot be fully studied without the sleep-state, or reverse.

\section{References}

Bagley EJ, El-Sheikh M (2013) Familial risk moderates the association between sleep and zBMI in children. J Pediatr Psychol 38:775-784

Ding D, Rogers K, Macniven R, Kamalesh V, Kritharides L, Chalmers J et al. (2013) Revisiting lifestyle risk index assessment in a large Australian sample: Are sedentary behavior and sleep correlated with health outcomes? Prev Med. doi:10.1016/j.ypmed.2013.12.021

Kang SG, Lee YJ, Kim SJ, Lim W, Lee HJ, Park YM et al. (2014) Weekend catch-up sleep is independently associated with suicide attempts and self-injury in Korean adolescents. Compr Psychiatry 55(2):319-325

Keller PS, Kouros CD, Erath SA, Dahl RE, El-Sheikh M (2014) Longitudinal relations between maternal depressive symptoms and child sleep problems: the role of parasympathetic nervous system reactivity. J Child Psychol Psychiatry 55(2):172-179

Lamarche LJ, De Koninck J (2007) Sleep disturbance in adults with posttraumatic stress disorder: a review. J Clin Psychiatry 68:12571270

Mansukhani MP, Kolla BP, Surani S, Varon J, Ramar K (2012) Sleep deprivation in resident physicians, work hour limitations, and related outcomes: a systematic review of the literature. Postgrad Med 124: 241-249

Pigeon WR, Pinquart M, Conner K (2012) Meta-analysis of sleep disturbance and suicidal thoughts and behaviors. J Clin Psychiatry 73: e1160-e1167

Reed DA, Fletcher KE, Arora VM (2010) Systematic review: association of shift length, protected sleep time, and night float with patient care, residents' health, and education. Ann Intern Med 153:829-842

Singareddy RK, Balon R (2002) Sleep in posttraumatic stress disorder. Ann Clin Psychiatry 14:183-190

Spruyt K, Gozal D (2012) A mediation model linking body weight, cognition, and sleep-disordered breathing. Am J Respir Crit Care Med 185:199-205

Spruyt K, Molfese DL, Gozal D (2011) Sleep duration, sleep regularity, body weight, and metabolic homeostasis in school-aged children. Pediatrics 127:e345-e352 